\title{
Update on the appropriate use of linezolid in clinical practice
}

\author{
Roberto Manfredi \\ Department of Clinical and \\ Experimental Medicine, Division of \\ Infectious Diseases, "Alma Mater \\ Studiorum" University of Bologna, S. \\ Orsola-Malpighi Hospital, Bologna, \\ Italy
}

\begin{abstract}
Multi-antibiotic resistant Gram-positive cocci, which include Staphylococcus aureus, the coagulase-negative staphylococcal group, Enterococcus faecalis and Enterococcus faecium, and other streptococci, represent emerging pathogens especially in the setting of the immunocompromised, hospitalized patients, in particular when surgery, invasive procedures, or prosthetic implants are of concern, patients are admitted in intensive care units, or underlying chronic disorders and immunodeficiency are of concern, and broad-spectrum antibiotics or immunosuppressive drugs are widely administered. During the recent years, the phenomenon of multiresistant Gram-positive cocci is spreading to the community, where the retrieval of such microorganism is progressively increasing. The spectrum of available antimicrobial compounds for an effective management of these relevant infections is significantly impaired in selection and clinical efficacy by the emerging and spread of methicillin-resistant and more recently glycopeptide-resistant Gram-positive microbial strains. The first oxazolidinone derivative linezolid, together with the recently licensed quinupristin-dalfopristin, daptomycin, and tigecycline, followed by a number of glycopeptides, fluoroquinolones, and other experimental compounds on the pipeline, represent an effective response to the great majority of these concerns, due to their innovative mechanisms of action, their maintained or enhanced activity against multiresistant pathogens, their effective pharmacokinetic/pharmacodynamic properties, their frequent possibility of synergistic activity with other compounds effective against Gram-positive pathogens, and a diffuse potential for a safe and easy administration, also when compromised patients are of concern. The main problems related to the epidemiological and clinical features of multiresistant Gram-positive infection, the potential clinical indications of all recently available compounds compared with the standard of care of treatment of resistant Gram-positive infections, and updated data on efficacy and tolerability of linezolid as the golden standard compound for vancomycin-resistant Gram-positive cocci in multiple clinical situations, are outlined and updated on the ground of an extensive review of all the available, recent evidences coming from the international literature.
\end{abstract}

Keywords: resistant Gram-positive organisms, staphylococci, enterococci, oxazolidinones, linezolid

\section{The rising problem of multiresistant Gram- positive cocci Involved microorganisms and risk factors}

Since the early eighties, a significant reversal of tendency was observed especially in hospital settings, characterized by a predominance of Gram-positive over Gramnegative bacteria (which represented the most relevant concern in the two prior decades) (Karchmer 2000; Stevens et al 2004; Cunha 2005; Kollef 2005; Menichetti 2005). The increased life expectancy of the general population, the extended survival of patients with underlyng immunodeficiency and/or chronic disorders, the advances of surgical techniques and those of invasive diagnostic and therapeutic procedures and bone marrow and solid organ transplantation, the diffusion of the implant of 
prosthetic materials and other biocompatible materials, and the increased resort to endovascular catheters and devices with massive resort to parenteral nutrition techniques, all of them representing emerging risk factors for a massive bacterial colonization, which unavoidably turns into an increased risk of local and systemic infection. At the same time, the increased and prolonged administration of broad spectrum antimicrobial agents and their associations, and the wide employment of potent antimycotic agents, as therapeutic and/or prophylactic measures directed to manage the immunocompromised or otherwise at risk host, extensively contribute to the re-emerging of Gram-positive pathogens and their increasing resistance rates, especially via prolonged hospital admissions. Among these microorganisms, both coagulase-positive and coagulasenegative Staphylococci, and Enterococci, became of particular concern in the hospital environment because of their rising frequency, the severity of associated diseases, and the unpredictable spectrum antimicrobial extend resistance (Wenzel and Edmond 1998; Karchmer 2000; Linden 2002; Cunha 2005; Kollef 2005; Menichetti 2005; Segreti 2005; Pace and Yang 2006). This is also increasingly true in the pediatric population, as recently confirmed by the literature (Cunha 2005; Ladhani and Garbash 2005; Marcinak and Frank 2006).

Finally, the progressive shortening of hospitalization favors the spread of hospital-acquired, nosocomial pathogens into the community, where there is increasing concern for the rising retrieval of microorganisms with a resistance pattern similar to that observed at the hospital, which may be responsible for an increasing failure of initial, empiric therapeutic lines, when antimicrobial agents are administered before obtaining culture and in vitro resistance testing, or when cultures and antimicrobial susceptibility testing are pending or not available (Cunha 2005; Ellis and Lewis 2005; Menichetti 2005; Marcinak and Frank 2006; Pace and Yang 2006).

Together with the ever-evolving modification of the above-mentioned multiple environmental conditions, the changing features of both host and its microbial flora, and the broadened spectrum of currently available antiinfective compounds, other emerging features become prominent, related to the pathomorphism of clinical features of a number of infections caused by Gram-positive pathogens, often those which share an evident potential for the development and spread of antimicrobial resistance. When considering Staphylococci, the major pathogen Staphylococcus aureus steadily ranks at the first place among the majority of hospital-acquired pathogens, and novel syndromes characterized by the predominant role of bacterial toxemia are progressively emerging: the so-called staphylococcal scalded skin syndrome (or SSSS) is the paradigmatic clinical picture. Moreover, severe staphylococcal skin and soft tissue lesions have been increasingly attributed to the action of the staphylococcal Panton-Valentine leukocidin (Marcinak and Frank 2006). Remarkable levels of morbidity and mortality are also attributed to coagulase-negative cocci (ie, Staphylococcus epidermidis and related organisms), especially when vascular or bone-joint prosthetic devices, central vascular lines, ventilator-associated pneumonia, and total parenteral nutrition are of concern. Until a couple of decades ago, the same coagulase-negative staphylococci were usually considered as part of the normal saprophytic human flora, or trivial local contaminants. On the other hand, while streptocococcal scarlet fever, and post-streptococcal complications like rheumatic disease and acute glomerulonephritis virtually disappeared in the past two decades, a number of other disorders caused by highly pathogenic Streptococci gained increasing frequency and importance in the last decades, including severe cellulites, necrotizing fasciitis, and the so-called toxic shock syndrome (TSS).

\section{Incidence of severe Gram-positive infections caused by multiresistant pathogens}

In the industrialized countries of the planet, the rate of methicillin (oxacillin) resistance of Staphylococci among hospitalized patients may overcome $20 \%-25 \%$ of cases, with an extremely elevated frequency registered in specialized intensive care units and bone marrow and solid organ transplant units (Edmond et al 1999; Karchmer 2000; Manfredi et al 2004; Cunha 2005; Kollef 2005; Segreti 2005). A relevant multicentre survey of nosocomial bacteremia carried out in 49 hospitals of the US during three consecutive years, allowed the authors to recognize a $64 \%$ prevalence of Gram-positive pathogens, among over 10000 identified microorganisms (Edmond et al 1999). When analyzing the frequency of each single microorganisms in this study, coagulase-negative Staphylococci (32\%) preceded $S$. aureus (16\%), and Enterococci as a whole (11\%). The overall level of methicillin resistance ranked around $29 \%$ of isolated organisms, with peaks reaching $80 \%$, when coagulase-negative Staphylococci were specifically considered (Edmond et al 1999). 
In a $S$. aureus prevalence study carried out in Spain, an overall increase in resistance to the majority of antimicrobial agents was detected, mainly involving oxacillin (from a frequency ranging from $1.5 \%$ and $32.5 \%$ in the year 1986 , to $31.2 \%$ and $61.3 \%$ respectively in the year 2002 ), although all isolates remained susceptible to all the available glycopeptides, quinupristin-dalfopristin, and linezolid, and a surprisingly low resistance rate was found when examining cotrimoxazole ( $0.5 \%$ to $2.1 \%$ ) (Cuevas et al 2004). Also other studies noticed this somewhat elevated cotrimoxazole susceptibility (Bishara et al 2003; Roberts and Chambers 2005).

A similar phenomenon was observed at a large teaching hospital in Taiwan, where the noticeable rise of methicillinresistant Staphylococci and vancomycin-resistant Enterococci paralleled the increased prescription of glycopeptides, broad-spectrum beta-lactams, carbapenems, and fluoroquinolones, during recent years (Hsueh et al 2005).

Among these microorganisms which share a predominant nosocomial isolation, the appearance of methicillin resistance is usually linked to an almost complete lack of in vitro susceptibility to all beta-lactam antibiotics, but usually it extends to macrolides, lincosamides, and a large part of aminoglycosides and fluoroquinolones, therefore leading to a very striking reduction of remaining therapeutic options (Wenzel and Edmond 1998; Lamb et al 1999; Karchmer 2000; Linden 2002; Stevens et al 2004; Cunha 2005; Kollef 2005; Menichetti 2005; Segreti 2005; Pace and Yang 2006). Notwithstanding that the frequently concurrent pathophysiological conditions can also act unfavorably, by limiting both microbiological and clinical efficacy of an antimicrobial treatment in vivo (Table 1), however the selection and spread of pharmacoresistant bacterial strains represents the main feature responsible for their greatly reduced activity in current clinical practice.

\section{Guidelines for the management of resistant Gram-positive infections, and emerging of glycopeptide resistance}

The present recommendations for empiric antimicrobial chemotherapy of the immunocompromised and/or neutropenic patient take into careful account of the ethiological shift from Gram-negative towards Grampositive organisms which occurred during the past 20 years. In the past decade, the guidelines for empiric antibiotic therapy of at-risk patients strongly recommended the adjunct of drugs which are highly active against methicillin-resistant Gram-positive cocci, but concomitantly contributed to the appearance and further spread of mutant microbial strains, which may test either "intermediate" or are even "resistant" to both available glycopeptides (vancomycin and teicoplanin). In fact, these last compounds (vancomycin and teicoplanin) represented the "gold-standard" therapeutic reference for the management of multiresistant Grampositive cocci, until a few years ago (Karchmer 2000; Linden 2002; Manfredi et al 2004; Stevens et al 2004; Akins 2005; Cunha 2005; Kollef 2005; Menichetti 2005; Segreti 2005; Pace and Yang 2006). However, an extensive Italian survey over 1997-1998 (Nicoletti et al 2000), showed that Grampositive microorganisms were responsible for slightly more than $50 \%$ of respiratory infection and septicemia identified in three different Italian intensive care units, with absolute predominance of $S$. aureus $(29.2 \%)$, followed by coagulasenegative Staphylococci (9.5\%), Streptococcus pneumoniae (4.1\%), and Enterococcus faecalis (2.9\%). Methicillinresistance levels tested around $46 \%$ for $S$. aureus, but rose to $64 \%$ for coagulase-negative Staphylococci (Nicoletti et al 2000).

When evaluating the episodes of nosocomial sepsis, the international "SCOPE" study carried out in 1998 by the SCOPE Hospital Study Group (Marshall et al 1998) attributed the greatest incidence to coagulase-negative Staphylococci, followed by $S$. aureus and Enterococcus spp., whose mortality rates proved $21 \%, 25 \%$, and $32 \%$ of reported cases, respectively. Enterococcus spp. organisms showed an antimicrobial susceptibility pattern notably different between E. faecalis and E. faecium: this last pathogen (ie, E. faecium) was increasingly identified during recent years in association with methicillin resistance levels often greater than $50 \%$ of tested strains. Concurrently, the incidence of methicillin resistance among coagulasenegative Staphylococci (with S. epidermidis always representing the leading organism of the group), tested even greater in frequency (also over $60 \%-80 \%$ in different clinical settings) (Pace and Yang 2006).

Finally, an fierce discussion is still ongoing regarding the role and frequency of staphylococcal strains which test "intermediate" to vancomycin and glycopeptides in general (the so-called "glycopeptide-intermediate S. aureus" [GISA]), which were identified in Japan in 1996 (Anonymous 1997). The incidence of this last phenomenon is estimated to be still geographically contained, although it is more commonly recognized in countries where the frequent resort to the administration of glycopeptide 
Table I Factors which contribute at various levels to the choice of empirical or selective antimicrobial chemotherapy, when resistant Gram-positive cocci may be of concern

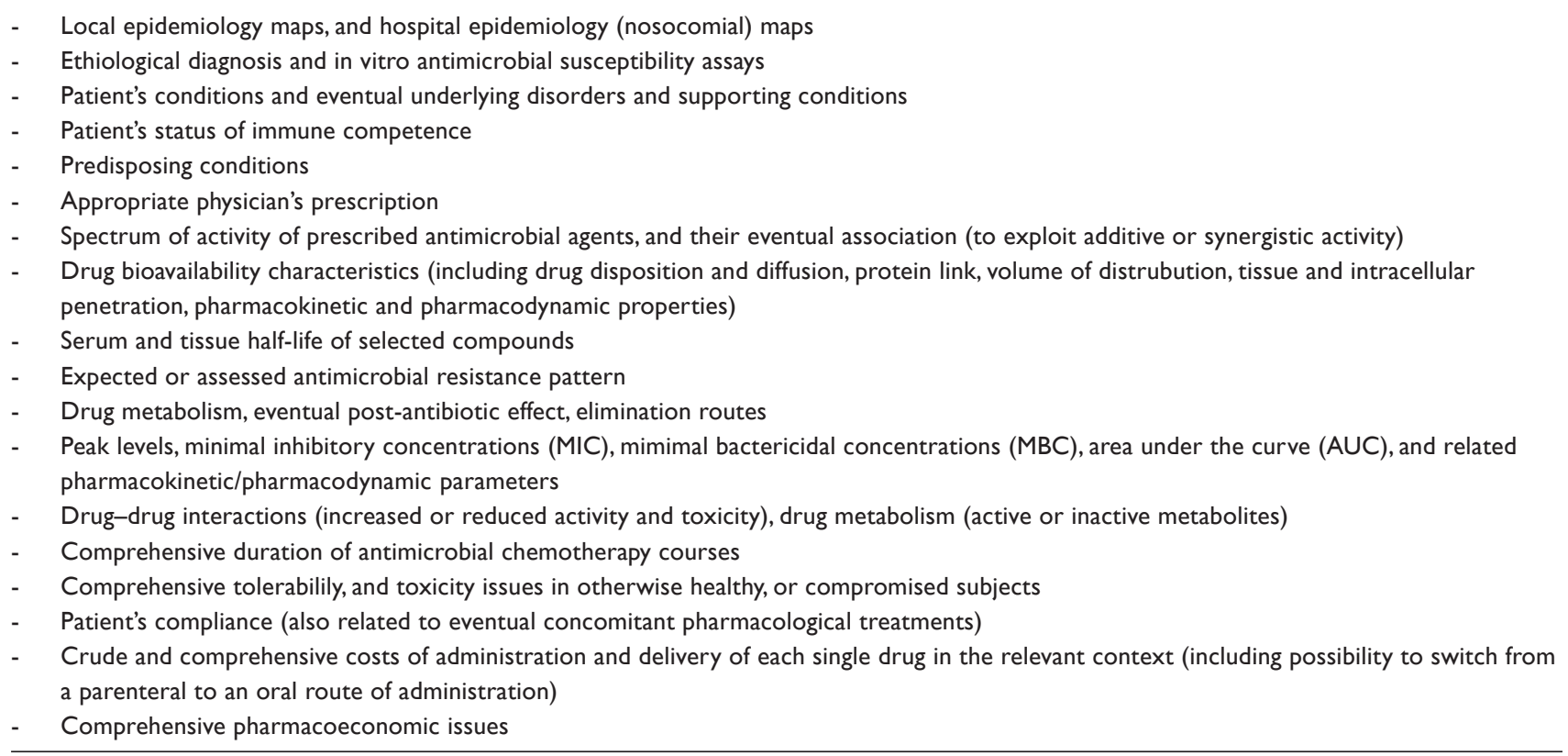

antibiotics supported a non-specific selective pressure over the relevant microorganims. The spread of glycopeptide (vancomycin-teicoplanin) resistance may occur through different bacterial species especially in the gastrointestinal tract, which may act as a reservoir of genes conferring resistance to glycopeptides and concurrently to many other antimicrobial compounds (Lipski et al 2004; Stevens et al 2004; Menichetti 2005; Segreti 2005; Pace and Yang 2006).

\section{The potential contribution of the novel oxazolidinone derivative: linezolid \\ Selected pharmacological issues}

Linezolid represent the first member of a novel class of oxazolidinone derivatives ( $a, q)$, which encompasses an effective activity spectrum which covers all the most important Gram-positive organisms, including those resistant to methicillin and glycopeptides. The chemical structure of linezolid is sketched in Figure 1. The oxazolidinones have an unique mode of action, which inhibits the initiation of the synthesis of bacterial proteins and enzymes, by preventing the formation of the ternary complex at 70S ribosomal subunit (Stevens et al 2004), by an apparent double blockade of both the 50S and the 30S bacterial ribosomal subunits. The uniquely particular mechanisms of action of linezolid, which includes a blockade of ribosomal assemblation which occurs before the initiation of bacterial protein synthesis (Stevens et al 2004), makes very improbable the emerging of cross resistance with other antimicrobial compounds. Single episodes of linezolid resistance have until now been anecdotally reported, especially after long-term and lowdosage courses, although it appears to be extremely rare among Staphylococci (Herrero et al 2002; Meka and Gold 2004), while linezolid-resistant Enterococci have been occasionally reported in intensive care units (Weigelt et al 2005), reports for both are now increasingly common.

From a pharmacokinetic point of view, linezolid is protein-bound for around $30 \%$, and this molecule favorably penetrates at elevated and fully active concentrations into a broad variety of tissues, such as fat, bone and joints, muscle, cerebrospinal fluid and wound sites, beyond the known, elevated penetration into the lungs and the entire respiratory tract (Eliopoulos 2003; Stevens et al 2004). The drug

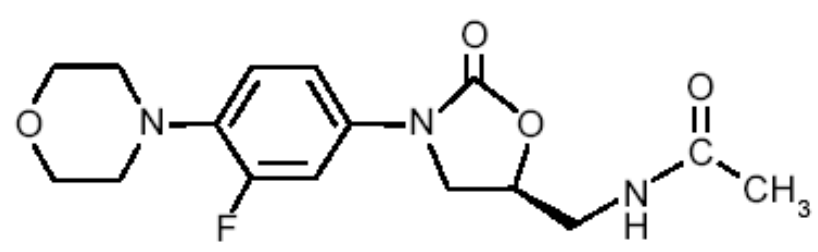

Figure I Chemical structure of linezolid, the first oxazolidinone antimicrobial compound. The first ring (from the left) contains a morpholino group, which enhances its pharmacokinetic profile and improves water solubility. In the second ring (from the left), the strategically located fluorine atom enhances drug activity. The "bridge" structure after the third ring (from the left) bears a necessary 5-(S) configuration. The terminal (right) C-5 acylaminomethyl group is essential for ensuring the unique drug characteristics. 
metabolism is not affected by the cytochrome $\mathrm{P} 450$ pathway, so that drug-drug interactions at this setting are not expected. Some selected pharmacokinetic properties of linezolid when administered intravenously compared with oral route (at standard dosages) are listed in Table 2.

Being formulated for both the intravenous (IV) and the oral route of administration, linezolid retains a $100 \%$ bioavailability even after oral administration (Eliopoulos 2003; Stevens et al 2004), and regardless of meals, therefore making it easier to exploit the oral administration for switching therapies and early discharge from the hospital of patients who can be effectively followed on a DayHospital outpatient basis, and also to directly start antibacterial therapy by oral route, whenever possible. All the above-mentioned aspects have a significant amount of favorable consequences, when comprehensive morbidity and mortality rates, overall length of hospital stay, and increased medical expenses are considered, even when compared with the proportionally elevated costs attributable to linezolid therapy (Moellering 2003; Stevens et al 2004).

\section{Microbiological properties, clinical studies, and clinical indications}

After expanded-access studies which included seriously ill patients suffering from multidrug-resistant, Gram-positive infections in situations also encompassing bacteremia (46\% of 796 cases), endocarditis, and catheter-related infection (Birmingham et al 2003), at its first approval in the US, linezolid was initially registered for the treatment of both community-acquired and nosocomial pneumonia, uncomplicated and complicated skin and soft tissue infections (including diabetic foot infections and surgical wound infection), and infectious caused by methicillin- and vancomycin-resistant Staphylococci and Enterococci, and penicillin-resistant and macrolide-resistant Streptococci and Pnemococci, including episodes complicated by bacteremia (Moellering 2003).

Since ribosomal mutations have been detected which may induce resistance against linezolid, longitudinal surveillance surveys remain needed to strictly monitor this phenomenon. The 2003 Zyvox Annual Appraisal of Potency and Spectrum (ZAAPS) program, compared the $\mathrm{MIC}_{90}$ levels of linezolid with 13-15 comparator agents in over 8000 bacterial isolates (Anderegg et al 2005), and confirmed a maintained $99.93 \%$ linezolid susceptibility rate of tested Gram-positive organisms, as previously predicted (Meka and Gold 2004). The recently published continuation of this
Table 2 Mean multiple-dose pharmacokinetics of linezolid, administered twice daily, as recommended (modified from Stevens et al 2004)

\begin{tabular}{lll}
\hline Parameter & $\mathbf{6 0 0} \mathbf{~ m g ~ o r a l ~ t a b l e t}$ & $\mathbf{6 0 0} \mathbf{~ m g ~ I V}$ \\
\hline $\mathrm{C}_{\max }(\mathrm{mcg} / \mathrm{mL})$ & $21.2 \pm 5.78$ & $15.10 \pm 2.52$ \\
$\mathrm{C}_{\min }(\mathrm{mvg} / \mathrm{mL})$ & $6.15 \pm 2.94$ & $3.68 \pm 2.36$ \\
$\mathrm{~T}_{\max }$ (hours) & $1.03 \pm 0.62$ & $0.51 \pm 0.03$ \\
$\mathrm{AUC}(\mathrm{mcgh} / \mathrm{mL})$ & $138.0 \pm 42.1$ & $89.7 \pm 31.0$ \\
Half-life (hours) & $5.40 \pm 2.06$ & $4.80 \pm 1.70$ \\
Clearance $(\mathrm{mL} / \mathrm{min})$ & $80.0 \pm 29.0$ & $123.0 \pm 40.0$ \\
\hline
\end{tabular}

Abbreviations: AUC, area under the curve.

study until 2004 (Jones et al 2006) confirmed this figure on even 20158 overall tested isolates, pointing out that $99.5 \%$ of isolated $S$. aureus organisms had a $\mathrm{MIC}_{90}$ value of linezolid ranging from $0.5 \mathrm{mg} / \mathrm{L}$ to $2 \mathrm{mg} / \mathrm{L}$, with only one bacterial strain tested susceptible only at a $\mathrm{MIC}_{90}$ value of $4 \mathrm{mg} / \mathrm{L}$ (Jones et al 2006).

In particular, when considering complicated skin and soft tissue infections, a randomized, open study has been conducted on 1200 hospitalized patients with confirmed or suspected methicillin-resistant staphylococcal isolates (Sharpe et al 2005). The most common disorders were represented by severe cellulitis $(46 \%)$, cutaneous abscess (26\%), and surgical wound infection (11\%). In the intentionto-treat comparison between linezolid (given at $600 \mathrm{mg}$ IV twice daily) and vancomycin (at $1 \mathrm{~g}$ IV twice daily), the cure rate was $92.2 \%$ versus $88.5 \%$ respectively $(p=0.057)$, while involed pathogens included methicillin-resistant Staphylococci in $42 \%$ of cases, followed by methicillinsensitive Staphylococci (29\%), and coagulase-negative Staphylococci (8\%) (Sharpe et al 2005). When considering methicillin-resistant staphylococcal infections only, a greater percentage of success rate was obtained with linezolid over vancomycin (88.6\% vs $66.9 \%$ respectively; $\mathrm{p}<0.001)$, paralleling the better bacteriological success rate of linezolid over vancomycin $(\mathrm{p}<0.001)$ (Sharpe et al 2005). In a subset of the above-mentioned study including surgical site infections presumably due to methicillin-resistant Staphylococci, although the clinical cure rate proved similar, linezolid obtained a greater bacteriological eradication rate $(\mathrm{p}<0.008)$, and a higher rate of microbiological cure $(\mathrm{p}<0.003)$ (Sharpe et al 2005). In an open study of complicated staphylococcal skin and soft tissue infections, oral linezolid and IV vancomycin were compared at the same dosage regimens for 7-21 days, and a higher clinical response was obtained with linezolid $(p<0.02)$, associated with a lower rate of failure leading also to a resort to major 
surgical procedures, ie, limb amputation $(\mathrm{p}<0.02)$ (Rubinstein et al 2001). Later, Weigelt and colleagues (2005) assessed linezolid versus vancomycin in the treatment of complicated skin and soft tissue infections in a randomized trial, but an evident overall superiority of linezolid was not reached from a statistical point of view.

Moreover, lower respiratory infections and pneumonia were assessed in two large, multicentre, randomized, doubleblind trials conducted on 1019 patients on the whole, affected by a nosocomial pneumonia presumably due to Gram-positive organisms (Wunderink et al 2003; Wilcox et al 2004). In these studies, an empirical linezolid treatment proved comparable with a vancomycin one, from both an efficacy and safety point of view (Wunderink et al 2003; Wilcox et al 2004). In a subgroup which included 123 patients with a confirmed hospital-acquired methicillinresistant staphylococcal pneumonia, a greater survival rate ( $84 \%$ vs $62 \%$; $\mathrm{p}=0.02)$, and clinical cure ( $62 \%$ vs $21 \%$; $\mathrm{p}<0.001)$ were achieved with linezolid, compared with vancomycin. In another study (Nathwani et al 2004) which compared the efficacy of linezolid and teicoplanin in 430 patients with differently located, ascertained or presumed Gram-positive infections, linezolid proved as effective as teicoplanin in patients suffering from pneumonia $(96 \%$ vs 93\%), but infections complicated by bacteremia had a greater response rate when linezolid was administered $(88.5 \%$ vs $56.7 \% ; p<0.001)$ (Nathwani et al 2004). Finally, the increased and proven recognition of the clinical potential of linezolid led to the inclusion of this novel oxazolidinone derivative in the treatment guidelines of the American Thoracic Society and those of the Infectious Disease Society of America (IDSA), as an empirical, initial choice for patients with a suspected nosocomial pneumonia caused by methicillin-resistant Staphylococci, or where the local, global prevalence of these organisms is known to be elevated (Moellering 2003; Craven et al 2004).

In adjunct, smaller patient series and anecdotal case reports of linezolid use in other relevant infectious processes are increasing day by day. The excellent tissue penetration of linezolid makes this oxazolidinone drug extremely promising for the approach to difficult-to-treat endocarditis with or without bacteremia (Hill et al 2006), central nervous system infections (Castro et al 2005; Sabbatani et al 2005; Manfredi et al 2006), and bone and joint infections (Manfredi et al 2005; Barberan 2006), caused by resistant Gram-positive cocci. Randomized clinical experiences are strongly expected to enforce eventual linezolid indications in these difficult-to-treat infections.
In the meantime, also pediatric experiences with linezolid are increasing day by day (Eliopoulos 2003; Ladhani et al 2005; Marcinak and Frank 2006).

A combination therapy of linezolid with other antimicrobial compounds is indicated when the co-existence of Gram-negative pathogens is ascertained or suspected. Moreover, a very interesting activity of linezolid has been demonstrated both in vitro and in vivo against susceptible and especially multi-drug resistant Mycobacterium tuberculosis (Alcala et al 2003; Erturan and Uzun 2005; Fortun et al 2005), and a synergistic activity may be exploited with a broad spectrum of fluoroquinolones (Rodriguez Diaz et al 2003; Barberan 2006), although the clinical significance of those associations needs to be clinically confirmed by controlled clinical trials.

\section{Tolerability and safety issues}

From a tolerability point of view, within the maximum allowed treatment duration of 28 days (as presently recommended), linezolid showed a favorable safety profile, as showed in a very extensive meta-analysis of 2046 adult patients enrolled in seven different comparative, controlled studies (Moellering 2003; Bressler et al 2004). The $85 \%$ of reported adverse events was mild-to-moderate in intensity: the most common clinical events were diarrhea $(4.0 \%$ to $5.3 \%$ ), nausea ( $3.3 \%$ to $3.5 \%$ ), and headache ( $1.9 \%$ to $2.7 \%$ ), while the most common laboratory disturbances included anemia and thrombocytopenia, especially when linezolid treatment is prolonged, as in some experimental indications, like bone and joint infections (Moellering 2003; Bressler et al 2004; Craven et al 2004; Senneville et al 2005).

During the post-marketing surveillance, sparse cases of neuritis (also including sparse cases of optical neuritis), and lactic acidosis were anecdotally reported, especially when linezolid treatment was extended beyond four weeks (Nasraway et al 2003; Kopterides et al 2005). Also myelotoxic effects and thrombocytopenia (whose pathogenesis still remains unknown) were associated with prolonged treatment durations, although some reports showed a somewhat earlier appearance. However, the risk of clinically significant thrombocytopenia as assessed on 686 patients with nosocomial pneumonia treated with linezolid for at least five days was limited to $6.4 \%$ (compared with $7.7 \%$ observed in the comparative vancomycin group) (Moellering 2003; Salyers et al 2004; Stevens et al 2004). When chronic or acute kidney insufficiency or hemodyalisis or hemofiltration are of concern, no correction of linezolid dosage are needed (Fiaccadori et al 2004), as compared with 
the limitations which become necessary when glycopeptide antibiotics (vancomycin or teicoplanin) are administered (Eliopoulos 2003; Craven et al 2004). However, an accumulation of linezolid metabolites has been observed in patients with renal impairment. Therefore, the selection of linezolid appears more indicated when a co-existing renal insufficiency may hamper the use of glycopeptides and other drugs with an increased renal toxicity. Finally, the well known activity exerted by linezolid on the inhibition of monoamine oxidase may prompt potential adverse drugdrug interactions with a broad range of antidepressant medications, which has to be carefully taken into account when facing patients who receive complex, multiple pharmacological treatments (Clark et al 2006; Lawrence et al 2006; Sola et al 2006).

\section{Preliminary pharmacoeconomic assessments}

A relevant European study (Rubinstein et al 2003) considered 227 patients with serious Gram-positive infections, treated with linezolid compared with teicoplanin, and assessed hospital resource use and overall treatment costs. The enrolled patients were randomized according to a 50:50 ratio to receive either linezolid for 7 to 28 consecutive days (initially by IV route, but with the possibility to shift to the oral administration as soon as possible), or teicoplanin (initially by IV administration, potentially followed by intramuscular route) (Rubinstein et al 2003). The mean IV treatment duration was 3.2-days shorter in the linezolid group (6.3 vs 9.5 days), tending to lead to a reduction of overall hospitalization costs, when comparing the novel oxazolidinone linezolid even against the most expensive glycopeptide teicoplanin (Moellering 2003; Rubinstein et al 2003).

Although the efficacy and safety of linezolid have been well demonstrated and checked in serious, high-risk and life-threatening infections where multiresistant Grampositive cocci are involved, and multiple pharmacoeconomic appraisals show that the availability of a bioequivalent oral formulation and a rapid switch therapy from intravenous to oral route of administration, may effectively counterbalance the more elevated crude costs compared with older glycopeptides like vancomycin, however further controlled clinical trials are strongly needed to expand the indications of this promising antibiotic, and to check carefully its tolerability in more extensive patient populations and baseline conditions (ie, extreme life ages, comorbidity, drugdrug interactions, and so on) (Moellering 2003). A short summary of some key issues in the pharmacoeconomic evaluation of a costly drug address to save from lifethreatening infection is contained in Table 3.

A ponderate prescription of linezolid limited to selected cases of serious, resistant Gram-positive infections, associated with improved standards of control and monitoring of nosocomial infections, are expected to add significantly to the long-term planning of effective guidelines of prescription, and large-scale resource allocation, in the optimization process of the management of multiresistant Gram-positive infection in critical care settings.

\section{Need of confirmations and future developments}

The proportionally recent availability of linezolid and other novel agents devoted to the fight against glycopeptideresistant Gram-positive cocci (ie, quinupristin-dalfopristin, daptomycin, and others), determined significant changes in the scenario of the management of severe infections due to multiresistant Gram-positive pathogens, usually acquired at the hospital and by a somewhat immunocompromised host (Abbanat et al 2003; Akins et al 2005; Hancock 2005; Segreti 2005).

A recent, extensive survey conducted on 258 Grampositive bacterial organisms isolated from blood cultures at an US cancer reference centre allowed to compare for the first time the in vitro acitivity of daptomycin, linezolid, and quinupristin-dalfopristin (Smith et al 2005). Vancomycinresistant Enterococci represented the largest proportion of tested organisms $(32 \%)$, followed by methicillin-resistant coagulase-negative Staphylococci (23\%), and vancomycin-

Table 3 Pharmacoeconomic variables of antimicrobial agents to be evaluated, especially when when an agent burdened by a proportionally elevated crude cost has to be addressed (modified from Nathwani 2006)

OVERALL EXPENDITURES

- Direct

- Indirect

- Intangible ones

TIME HORIZON CONSIDERED

- Short-term

- Medium-term

- Long-term

DIFFERENT PERSPECTIVES

- Society

- Patient

- Payer

- Provider 
sensitive Enterococci (14\%). Through a detailed analysis of both MIC and mimimal bactericidal concentrations (MBC) values, daptomycin showed a bactericidal activity against the majority of tested organisms, by killing almost $100 \%$ of bacteria within six hours. Quinupristin-dalfopristin was bactericidal against Staphylococci and bacteriostatic against the majority of Enterococci. Linezolid was bacteriostatic against all evaluated organisms, but a correlation between the in vitro features and the clinical outcome demonstrated an elevated potential of all these novel compounds (Smith et al 2005), which now deserve controlled studies in the setting of the management and prevention of serious infection in the immunocompromised host, including HIV/AIDS patients, subjects with hematologic malignancies or solid tumors, and those undergoing bone marrow or organ transplantation, or major surgery and hospitalization in intensive care units. Interestingly, a recent study also pointed out a significant reduction of acute-phase proinflammatory cytokines from human peripheral mononuclear cells (Garcia-Roca et al 2006) when linezolid was administered, thus confirming an immunomodulating response to linezolid use.

\section{Conclusions and future developments and expectations}

The overall appraisal of the epidemiology and virulence of community-acquired multiresistant Gram-positive pathogens continue to grow, leading to major attention devoted to developing compounds, but also a re-examination of many older, but still active agents (including long-acting tetracyclynes, fluoroquinolones, rifampicin, cotrimoxazole, and clindamycin) (Moellering 2003; Craven et al 2004; Manfredi et al 2004; Shah 2005; Schmidt-Ioanas et al 2005), which certainly retain some non-negligible therapeutic role, especially when a synergistic activity can be demonstrated (Akins et al 2005; Ellis and Lewis 2005). Moreover, through novel laboratory assays like the so-called E-test synergy and time-kill methods will perhaps become possible to measure to extent of synergistic activity between differently combined molecules against glycopeptide-resistance grampositive cocci (ie, daptomycin and rifampicin against multiresistant Enterococci) (LaPlante and Rybak 2004; Hancock 2005; Shah 2005; Tsuji et al 2006).

As summarized above, future therapeutic research promises the development of novel compounds aimed at intervening favorably against the unavoidable increase of drug resistance frequency and levels against the present reference compounds (ie, the glycopeptides vancomycin and teicoplanin) (Pace and Yang 2006), and later the two abovementioned recent molecules, ie, quinupristin-dalfopristin and especially linezolid (Linden 2002; Abbanat et al 2003; Meka and Gold 2004; Akins et al 2005; Menichetti 2005; Schmidt-Ioanas et al 2005; Segreti 2005; Barberan 2006). Some relevant issues regarding linezolid, compared with the present standards of care (vancomycin and teicoplanin), and two recent comparators (quinupristin-dalfopristin and daptomycin), are summarized in Table 4.

On the other hand, the clinical use of the streptogramin combination quinupristin-dalfopristin, which retains

Table 4 Some key mode and resource use with antimicrobials specific for resistant Gram-positive infections. Linezolid compared with standard of care antimicrobials for methicillin-resistant Gram-positive cocci infections (vancomycin and teicoplanin), and with the currently available alternatives recently introduced into the market (quinupristin/dalfopristin, and daptomycin)

\begin{tabular}{|c|c|c|c|c|c|c|c|}
\hline & Linezolid & Vancomycin & Teicoplanin & $\begin{array}{l}\text { Quinupristin/ } \\
\text { dalfopristin }\end{array}$ & Daptomycin & Tigecycline & Dalbavancin \\
\hline $\begin{array}{l}\text { IV and oral route, } \\
\text { both available }\end{array}$ & Yes & No* & No* & No & No & No & No \\
\hline $\begin{array}{l}\text { Ambulatory } \\
\text { (outpatient) } \\
\text { administration } \\
\text { feasible }\end{array}$ & Yes & No & Yes & Yes? & ? & $?$ & Yes \\
\hline $\begin{array}{l}\text { Need of serum } \\
\text { drug level } \\
\text { monitoring } \\
\text { Frequency of drug }\end{array}$ & No & Yes & No & Yes & Yes? & No & No \\
\hline administration & Twice daily & $\begin{array}{l}\text { At least twice } \\
\text { daily, to } 24 \mathrm{~h} \\
\text { continue infusion }\end{array}$ & Once daily & Once daily & Once daily & Twice daily & Once weekly \\
\hline
\end{tabular}

Abbreviations: IV, intravenous.

Note: *indication restricted to the management of Clostridium difficile-associated diarrhea. 
optimal activity against methicillin-resistant $S$. aureus and vancomycin-resistant $E$. faecium, is limited because of its need to be administered in large volume of fluids (Shah 2005), while its activity in severe pneumonia is somewhat lower, therefore it is not strongly indicated (Manfredi et al 2004). The oxazolidinone linezolid is active against methicillin-resistant Staphylococci and glycopeptideresistant Enterococci, but resistant organisms and sparse treatment failures have been reported (Shah 2005), while unexpected, recent tolerability issues are becoming of concern (Kopterides et al 2005).

Finally, we have to remind that in many cases the most relevant therapeutic intervention for complicated Grampositive abscesses, cellulitis, complicated skin and soft tissue diseases, but also osteomyelitis, infected bone and joint prostheses, and brain abscesses, remains an adequate surgical drainage and curettage of purulent fluid collections, and the elimination of affected, necrotic tissue (Ellis and Lewis 2005; Shah 2005; Barberan 2006). Subsequently, the antimicrobial selection should be driven by disease severity, susceptibility patterns, clinical response to therapy, and also related costs (seen from a comprehensive point of view). Also special population such as the pediatric and neonatal ones (Lahdani et al 2005), are going to benefit from specifically-designed trials, which could address future issues in the setting of epidemiology, mechanisms of virulence, continued changes in pathogenicity and antimicrobial susceptibility of involved organisms, and potential use of novel antimicrobial compounds.

\section{References}

Abbanat D, Macielag M, Bush K. 2003. Novel antibacterial agents for the treatment of serious Gram-positive infections. Expert Opin Investig Drugs, 12:379-99.

Akins RL, Haase KK. 2005. Gram-positive resistance: pathogens, implications, and treatment options: insights from the Society of Infectious Diseases Pharmacists. Pharmacotherapy, 25:1001-10.

Alcala L, Ruiz-Serrano MJ, Pérez-Fernandez-Turégano C, et al. 2003. In vitro activities of linezolid against clinical isolates of Mycobacterium tuberculosis that are susceptible or resistant to first-line antituberculous drugs. Antimicrob Agents Chemother, 47:416-17.

Anderegg TR, Sader HS, Fritsche TR, et al. 2005. Trends in linezolid susceptibility patterns: report from the 2002-2003 worldwide Zyvox annual appraisal of potency and spectrum (ZAAPS) program. Int $J$ Antimicrob Agents, 26:13-21.

Anonymous. 1997. Reduced susceptibility of Staphylococcus aureus to vancomycin - Japan 1996. MMWR, 46:624-6.

Barberan J. 2006. Management of infections of osteoarticular prosthesis. Clin Microbiol Infect, 12(Suppl. 3):93-101.

Birmingham MC, Rayner CR, Meagher AK, et al. 2003. Linezolid for the treatment of multidrug-resistant, Gram-positive infections: experience from a compassionate-use program. Clin Infect Dis, 36:159-68.
Bishara J, Pitlik S, Samra Z, et al. 2003. Co-trimoxazole sensitive, methicillin-resistant Staphylococcus aureus, Israel, 1988-1997. Emerg Infect Dis, 9:1168-9.

Bressler AM, Zimmer SM, Gilmore JL, et al. 2004. Peripheral neuropathy associated with prolonged use of linezolid. Lancet Infect Dis, 4:52831.

Castro P, Soriano A, Eschrich C, et al. 2005. Linezolid treatment of ventriculoperitoneal shunt infection without implant removal. Eur $J$ Clin Microbiol Infect Dis, 24:603-6.

Clark DB, Andrus MR, Byrd DC. 2006. Drug interactions between linezolid and selective serotonin reuptake inhibitors: case report involving sertraline and review of the literature. Pharmacotherapy, 26:269-76.

Craven DE, Palladino R, McQuillen DP. 2004. Healthcare-associated pneumonia in adults: management principles to improve outcomes. Infect Dis Clin North Am, 18:939-62.

Cuevas O, Cercenado E, Vindel A, et al. 2004. Evolution of antimicrobial resistance of Staphylococcus spp. In Spain: five nationwide prevalence studies, 1986 to 2002. Antimicrob Agents Chemother, 48:4240-5.

Cunha BA. 2005. Methicillin-resistant Staphylococcus aureus: clinical manifestations and antimicrobial therapy. Clin Microbiol Infect, 11(Suppl. 4):33-42

Edmond MB Wallace SE, McClish DK, et al. 1999. Nosocomial bloodstream infections in United States hospitals. A three-year analysis. Clin Infect Dis, 29:239-44.

Eliopoulos GM. 2003. Quinupristin/dalfopristin and linezolid: evidence and opinions. Clin Infect Dis, 36:473-81.

Ellis MW, Lewis JS 2nd. 2005. Treatment approaches for communityacquired methicillin-resistant Staphylococcus aureus infections. Curr Opin Infect Dis, 18:496-501.

Erturan Z, Uzun M. 2005. In vitro activity of linezolid against multidrugresistant Mycobacterium tuberculosis isolates. Int J Antimicrob Agents, 26:78-80.

Fiaccadori E, Maggiore U, Rotelli C, et al. 2004. Removal of linezolid by conventional intermittent hemodyalisis, sustained low-efficiency dyalisis, or continuous venovenous hemofiltration in patients with acute renal failure. Crit Care Med, 32:2437-42.

Fortun J, Martin-Davilla P, Navas E, et al. 2005. Linezolid for the treatment of multidrug-resistant tuberculosis. J Antimicrob Chemother, 56:180-

Garcia-Roca P, Mancilla-Ramirez J, Santos-Segura A, et al. 2006. Linezolid diminishes inflammatory cytokine production from human peripheral blood mononuclear cells. Arch Med Res, 37:31-5.

Hancock RE. 2005. Mechanism of action of newer antibiotics for Grampositive pathogens. Lancet Infect Dis, 5:209-18.

Herrero IA, Issa NC, Patel R. 2002. Nosocomial spread of linezolidresistant, vancomycin-resistant Enterococcus faecium. $N$ Engl J Med, 346:867-9.

Hill EE, Herijgers P, Herregods MC, et al. 2006. Infective endocarditis treated with linezolid: case report and literature review. Eur J Clin Microbiol Infect Dis, 25:202-4.

Hsueh PR, Chen WH, Teng LJ, 2005. Nosocomial infections due to methicillin-resistant Staphylococcus aureus and vancomycin-resistant enterococci at a University Hospital in Taiwan from 1991 to 2003: resistance trends, antibiotic usage and in vitro activities of newer antimicrobial agents. Int J Antimicrob Agents, 26:43-9.

Jones RN, Ross JE, Fritsche TR, et al. 2006. Oxazolidinone susceptibility patterns in 2004: report from the Zyvox annual appraisal of potency and spectrum (ZAAPS) Program assessing isolates from 16 nations. J Antimicrob Chemother, 57:279-87

Karchmer AW. 2000. Nosocomial bloodstream infections: organisms, risk factors, and implications. Clin Infect Dis, 31(Suppl. 4):S139-43.

Kollef MH. 2005. Antibiotic management of ventilator-associated pneumonia due to antibiotic-resistant gram-positive bacterial infection. Eur J Clin Microbiol Infect Dis, 24:794-803.

Kopterides P, Papadomichelakis E, Armaganidis A. 2005. Linezolid use associated with lactic acidosis. Scand J Infect Dis, 37:153-4 
Ladhani S, Garbash M. 2005. Staphylococcal skin infections in children: rational drug therapy recommendations. Paediatr Drugs, 7:77-102.

Lamb HM, Figgitt DP, Faulds D. 1999. Quinupristin/dalfopristin: a review of its use in the management of serious gram-positive infections. Drugs, 58:1061-97.

LaPlante KL, Rybak MJ. 2004. Daptomycin - a novel antibiotic against Gram-positive pathogens. Exp Opin Pharmacother, 5:2321-31.

Lawrence KR, Adra M, Gillman PK. 2006. Serotonin toxicity with the use of linezolid: a review of postmarketing data. Clin Infect Dis, 42:1578-83.

Linden PK. 2002. Treatment options for vancomycin-resistant enterococcal infections. Drugs, 62:425-41.

Lipsky BA, Itani K, Norden C. 2004. Treating foot infections in diabetic patients: a randomized, multicenter, open-label trial of linezolid versus ampicillin-sulbactam/amoxicillin-clavulanate. Clin Infect Dis, 38:1724.

Manfredi R, Nanetti A, Morelli S, et al. 2004. Sensitivity of methicillinresistant Staphylococcus aureus (MRSA) to antimicrobial agents other than beta-lactams: profile and trend, 1998-2003. Isr Med Assoc J, 6:191-2.

Manfredi R, Sabbatani S, Chiodo F. 2005. Severe staphylococcal knee arthritis responding favourably to linezolid, after glycopeptiderifampicin failure: a case report and literature review. Scand J Infect Dis, 37:513-17.

Manfredi R, Sabbatani S, Marinacci G. 2006. Listeria monocytogenes meningitis and multiple brain abscesses in an immunocompetent host. Favorable response to combined linezolid treatment. $J$ Chemother, 18:331-3.

Marcinak JF, Frank AL. 2006. Epidemiology and treatment of communityassociated methicillin-resistant Staphylococcus aureus in children. Expert Rev Anti Infect Ther, 4:91-100.

Marshall SA, Wilke WW, Pfaller MA, et al. 1998. Staphylococcus aureus and coagulase-negative staphylococci from blood stream infections: frequency of occurrence, antimicrobial susceptibility, and molecular (mecA) characterization of oxacillin resistance in the SCOPE program. Diagn Microbiol Infect Dis, 30:205-14.

Meka VG, Gold HS. 2004. Antimicrobial resistance to linezolid. Clin Infect Dis, 39:1010-5

Menichetti F. 2005. Current and emerging serious Gram-positive infections. Clin Microbiol Infect, 11 (Suppl. 3):22-8.

Moellering RC Jr. 2003. Linezolid: the first oxazolidinone antimicrobial. Ann Intern Med, 138:135-42.

Nasraway SA, Shorr AF, Kuter DJ, et al. 2003. Linezolid does not increase the risk of thrombocytopenia in patients with nosocomial pneumonia: comparative analysis of linezolid and vancomycin use. Clin Infect Dis, 37:1609-16.

Nathwani D, Li JZ, Balan DA, et al. 2004. An economic evaluation of a European cohort from a multinational trial of linezolid versus teicoplanin in serious Gram-positive bacterial infections: the importance of treatment setting in evaluating treatment effects. Int $J$ Antimicrob Agents, 23:315-24.

Nathwani D. 2006. Personal communication.

Nicoletti G, Bonfiglio G, Bartoloni A, et al. 2000. Distribution and antibiotic resistance of isolates from lower respiratory tract and blood cultures from patients in three Italian intensive care units: a 2-year comparison. Int J Antimicrob Agents, 15:265-9.

Pace JL, Yang G. 2006. Glycopeptides: update on an old successful antibiotic class. Biochem Pharmacol, 71:968-80.
Roberts S, Chambers S. 2005. Diagnosis and management of Staphylococcus aureus infections of the skin and soft tissue. Intern Med J, 35(Suppl. 2):S97-105.

Rodriguez Diaz JC, Ruiz M, Lopez M, et al. 2003. Synergistic activity of fluoroquinolones and linezolid against Mycobacterium tuberculosis. Int J Antimicrob Agents, 21:354-6.

Rubinstein E, Cammarata S, Oliphant T, et al. 2001. Linezolid (PNU100766) versus vancomycin in the treatment of hospitalized patients with nosocomial pneumonia: a randomized, double-blind multicenter study. Clin Infect Dis, 32:402-12.

Rubinstein E, Isturiz R, Standiford HC, et al. 2003. Worldwide assessment of linezolid's clinical safety and tolerability: comparator-controlled Phase III studies. Antimicrob Agents Chemother, 47:1824-31.

Sabbatani S, Manfredi R, Frank G. 2005. Linezolid in the treatment of central nervous system infections resistant to recommended antimicrobial compounds. Infez Med, 13:112-19.

Salyers AA, Gupta A, Wang Y. 2004. Human intestinal bacteria as reservoirs for antibiotic resistance genes. Trends Microbiol, 12:412-16.

Schmidt-Ioanas M, De Roux A, Lode H. 2005. New antibiotics for the treatment of severe staphylococcal infection in the critically ill patient. Curr Opin Crit Care, 11:481-6.

Segreti J. 2005. Efficacy of current agents used in the treatment of Grampositive infections and the consequences of resistance. Clin Microbiol Infect, 11(Suppl. 3):29-35.

Senneville E, Legout L, Valette M, et al. 2005. Risk factors for anaemia in patients on prolonged linezolid therapy for chronic osteomyelitis: a case-control study. J Antimicrob Chemother, 56:440-1.

Shah PM. 2005. The need for new therapeutic agents: what is the pipeline? Clin Microbiol Infect, 11(Suppl. 3):36-42.

Sharpe JN, Shively EH, Polk HC Jr. 2005. Clinical and economic outcomes of oral linezolid versus intravenous vancomycin in the treatment of MRSA-complicated, lower extremity skin and soft tissue infections caused by methicillin-resistant Staphyloccus aureus. Am J Surg, 189:425-8.

Smith PF, Booker BM, Ogundele AB, et al. 2005. Comparative in vitro activities of daptomycin, linezolid, and quinupristin/dalfopristin against Gram-positive bacterial isolates from a large cancer center. Diagn Microbiol Infect Dis, 52:255-9.

Sola CL, Bostwick JM, Hart DA, et al. 2006. Anticipating potential linezolid-SSRI interactions in the general hospital setting: an MAOI in disguise. Mayo Clin Proc, 81:330-4.

Stevens DL, Dotter B, Madaras-Kelly A. 2004. A review of linezolid: the first oxazolidinone antibiotic. Expert Rev Anti-Infective Ther, 2:51-9.

Tsuji BT, Rybak MJ. 2006. Etest synergy testing of clinical isolates of Staphylococcus aureus demonstrating heterogeneous resistance to vancomycin. Diagn Microbiol Infect Dis, 54:73-7.

Weigelt J, Itani K, Stevens D, et al. 2005. Linezolid versus vancomycin in treatment of complicated skin and soft tissue infections. Antimicrob Agents Chemother, 49:2260-6.

Wenzel RP, Edmond MB. 1998. Vancomycin-resistant Staphylococcus aureus: infection control considerations. Clin Infect Dis, 27:245-9.

Wilcox M, Nathwani D, Dryden M. 2004. Linezolid compared with teicoplanin for the treatment of suspected or proven Gram-positive infections. J Antimicrob Chemother, 53:335-42.

Wunderink RG, Cammarata SK, Oliphant TH, et al. 2003. Continuation of a randomized, double-blind, multicenter study of linezolid versus vancomycin in the treatment of patients with nosocomial pneumonia. Clin Ther, 25:980-92. 